Texture of Crystalline Solids, 1980, Vol. 4, pp. 111-127

0390-7951/80/0402-0111\$04.50/0

() 1980 Gordon and Breach, Science Publishers, Inc.

Printed in the United States of America

\title{
ELASTIC PROPERTIES OF COLD-ROLLED AND ANNEALED SHEETS OF PHOSPHORUS STEEL HAVING HIGH NORMAL PLASTIC ANISOTROPY
}

\author{
HSUN HU \\ U. S. Steel Research Laboratory, \\ Monroeville, PA 15146 USA
}

(Received January 10, 1980)

\begin{abstract}
The elastic properties of as-cold-rolled and of the subsequently annealed sheets of a phosphorus steel having high normal plastic anisotropy and low planar plastic anisotropy have been calculated according to the averaging procedures of voigt, Reuss, and Hill incorporated with texture-weighting functions. The calculated values of Young's modulus in the various directtions lying in the plane of the sheet were compared with those determined experimentally by ultrasonic measurements. Results indicate that the Hill averages are very close to reality, whereas the Voigt and Reuss averages are somewhat too high and too low, respectively, in comparison with experimentally measured values. This paper has been prepared to help familiarize metallurgists, engineers, and students with the mathematical treatments that can be used for such studies. The procedures used for the calculations are described in detail, and examples of calculations are provided in the Appendix.
\end{abstract}

\section{INTRODUCTION}

In an earlier report ${ }^{1}$ the spatial-orientation distributions of the crystallites in cold-rolled and annealed sheets of a strongly (IIl) textured phosphorus steel having high normal plastic anisotropy (r value) and low planar plastic anisotropy ( $\Delta r$ value) were studied by the generalized spherical harmonics method. ${ }^{2-5}$ The orientation-distribution functions so obtained provide a quantitative and unambiguous representation of the textures in these materials. By using these orientation-distribution functions the cold-rolling texture and the annealing texture of the steel and the relationship between these textures can be studied in more detail. Physical or mechanical properties of textured sheets can also be calculated from the known property data for single crystals and appropriate texture-weighting functions. Moreover, such 
calculations not only predict the properties of texture materials, but also provide a theoretical understanding of how the properties of single crystals contribute to the properties of variously textured polycrystalline aggregates.

Elastic properties of textured sheets calculated from orientation-distribution functions have so far been reported only by a few investigators for some of the common nonferrous metals or alloys ${ }^{6-10}$ and for the low-carbon aluminum-killed steels.9,11 These publications, however, presented primarily the final results. The procedures or mathematical operations required to perform the calculations were not provided.

For applied mathematicians or experts in the field, the existing publications are adequate and informative. However, for most physical metallurgists or material scientists, such highly condensed publications involving sophisticated mathematics cannot be easily digested and thus will not be utilized. This situation is unfortunate, because material properties and their relations to structures are a major concern of physical metallurgists and material scientists.

In consideration of the above, the calculations of the elastic properties of sheets from a strongly (1ll) textured phosphorus steel ${ }^{1}$ will be presented step by step, and in more detail than in previously published calculations. It is hoped that the present report, besides providing basic information on the elastic properties of textured sheets of the phosphorus steel, will also be useful to researchers who wish to conduct similar studies but who have not known how to perform the mathematical operations.

\section{ELASTIC PROPERTIES OF POLYCRYSTALLINE AGGREGATES}

From single-crystal data, the elastic properties of polycrystaline aggregates can be calculated by an averaging scheme, with certain assumptions. The averaging procedure of Voigt ${ }^{12}$ assumed that the strain of all crystals in the aggregate was the same, whereas that of Reuss ${ }^{3}$ assumed uniform stress throughout the polycrystalline specimen. Hill, 14 however, considered neither of these two models to be truly realistic because in the constant-strain model the forces between neighboring grains would not likely be in equilibrium, whereas in the constant-stress model the distorted grains would result in discontinuities at the grain boundaries. Hill showed that the moduli as determined by Voigt will be greater than the moduli as determined by Reuss, whereas the true values should lie between them.

Each of the approximation methods mentioned above (VoigtReuss-Hill approximations) considered that the aggregate contains individual crystals of all orientations (random textured), and is macroscopically homogeneous. It was shown by Chung and Buessem, ${ }^{15}$ who examined a number of random polycrystalline aggregates of cubic compounds having various anisotropy parameters, that the calculated elastic moduli based on the Hill approximation agreed very well with the measured values when the crystals were not highly anisotropic. A more sophisticated treatment for the calculation of the effective 
elastic moduli of randomly oriented polycrystalline aggregates was conducted by Kröner, ${ }^{16}$ who took the boundary conditions of the stresses and strains across the grain boundaries into account. However, the discrepancies between the results obtained with Kröner's method and those obtained with Hill's method were reportedly insignificantly small, being within the limits resulting from the uncertainty of the single-crystal elastic constants. 1 i

For the strongly textured sheets of the cold-rolled and of the subsequently annealed phosphorus steel, direct application of the Voigt-Reuss-Hill approximations for the elasticmoduli calculations would obviously be inappropriate.

Bunge 5,17 showed how to generalize the Voigt-Reuss-Hill approximations to apply to textured polycrystalline aggregates of cubic crystals. Morris ${ }^{18}$ developed the method of averaging fourth-rank tensors with texture-weighting functions for sheet materials having orthorhombic and higher (such as the tetragonal, hexagonal, and cubic) crystal symmetries. The coefficients of the generalized spherical harmonics series, which quantitatively specify the textures, are used in the weighting functions. The present report describes the method and results of calculations as formulated by Morris, 18 and compares the calculated elastic properties of the strongly textured cold-rolled and annealed sheets of the phosphorus steel with those obtained from experimental measurements.

\section{The Weighted Voigt Average}

Because the Voigt averaging scheme assumes constant strain throughout the aggregate, the calculations will naturally be performed for the averaged elastic stiffness constants, $\bar{c}_{i j k l}$, of the aggregate by using the elastic stiffness constants of a single crystal. As shown by Morris, 9, 18 the weighted average $\bar{c}_{i j}$ in Voigt two-index notation (see, for example, Reference 19 for the index notation of elastic constants) is given by

$$
\left[\bar{c}_{i j}\right]=\left[\bar{T}_{i j k \ell}\right]\left[c_{k \ell}\right]
$$

where $\overline{\mathbf{c}}_{i j}$ are the weighted stiffness constants of the aggregate, which for orthotropic specimen symmetry (rolled sheet) comprise nine constants having indices $i j=11,22,33,23$, $31,12,44,55$, and 66 . The $c_{k l}$ are stiffness constants of the single crystal.

In connection with Equation (1), some explanation for the meaning of the expression may be desired. It is important to note that the transformation rule used in the averaging procedure applies only to tensor components, i.e., $\bar{c}_{i j k \ell}=\bar{T}_{i j k l m n p q} c_{m n p q}$, where a repeated subscript implies summation over $1,2,3$. In order to be concise, those $\bar{T}_{i j k \ell m n p g}$ for which ' $c_{m n p q}$ are equivalent may be combined, and the result expressed in an abbreviated four-index notation, such as $\overline{\mathrm{T}}_{1111}=\overline{\mathrm{T}}_{11111111,}, \overline{\mathrm{T}}_{1112}=\overline{\mathrm{T}}_{11111122}+\overline{\mathrm{T}}_{11112111}$,

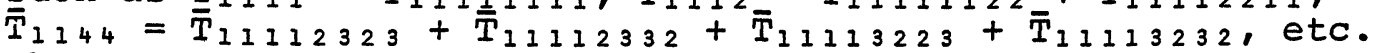
The presence of equivalent cmnpq due to crystal symmetry leads 
additional combinations. $\overline{\mathrm{T}}_{1111}$ (cubic) $=\overline{\mathrm{T}}_{11111111+}$

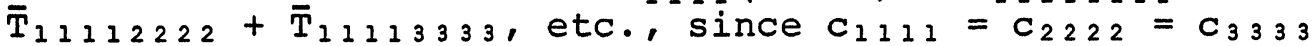
for cubic crystal symmetry. For cubic crystal symmetry, the expression $\bar{c}_{11}=\bar{T}_{11 k l} c_{k l}$ is intended to imply:

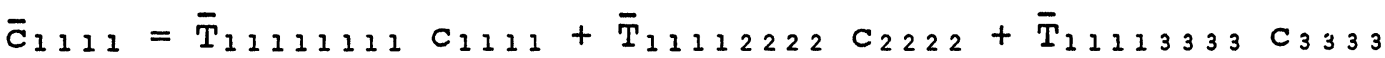

$$
\begin{aligned}
& +\bar{T}_{11112233} c_{2233}+\bar{T}_{11113322} c_{3322}+\bar{T}_{11113311} c_{3311} \\
& +\bar{T}_{11111133} c_{1133}+\bar{T}_{11111122} c_{1122}+\bar{T}_{11112211} c_{2111} \\
& +\bar{T}_{11112323} c_{2323}+\bar{T}_{11112332} c_{2332}+\bar{T}_{11113223} c_{3223} \\
& +\bar{T}_{11113232} c_{3232}+\bar{T}_{11113131} c_{3131}+\bar{T}_{11113113} c_{3113} \\
& +\bar{T}_{11111331} c_{1331}+\bar{T}_{11111313} c_{1313}+\bar{T}_{11111212} c_{1212} \\
& +\bar{T}_{11111221} c_{1221}+\bar{T}_{11112112} c_{2112}+\bar{T}_{11112121} c_{2121}
\end{aligned}
$$

Because, for cubic crystal symmetry, $c_{1111}=c_{2222}=c_{33}{ }_{33}$, $\mathrm{C}_{2233}=\mathrm{C}_{3311}=\mathrm{C}_{1122}$, and $\mathrm{c}_{2323}=\mathrm{c}_{3131}=\mathrm{c}_{1212}$, therefore, we may write,

$$
\bar{c}_{11}=\bar{T}_{1111} c_{11}+\bar{T}_{1112} c_{12}+\bar{T}_{1144} c_{44}
$$

It is important to remember that use of the two-index notation (matrix) for the $c_{i j k l}$ and four-index notation for the $\bar{T}_{i j k l m n p q}$ is only a convenience, and that the relation applies to tensor components. This presents no difficulty in averaging elastic stiffnesses. When averaging elastic compliances, however, special cautions in the notation should be exercised to avoid confusion or misleading. These will be discussed in the next section when the weighted Reuss averages are calculated.

For cubic crystals, there are only three elastic constants with indices $\mathrm{kl}=11,12$, and 44 . Thus, for rolled sheet of iron and steel or any other cubic metals the $\overline{\mathrm{T}}_{i j k l}$ are the elements of a 9 by 3 matrix given by

$\overline{\mathrm{T}}_{i j k l}=\mathrm{A}_{000}+4 \pi^{2}\left(\mathrm{~A}_{400} \mathrm{~W}_{400}+\mathrm{A}_{420} \mathrm{~W}_{420}+\mathrm{A}_{440} \mathrm{~W}_{440}\right)$

where $W_{l m n}$ are coefficients of the spatial orientation distribution of the crystallites, and the values of Apqr depend on the indices ijkl. The $\bar{T}_{i j k l}$ values for cubic crystal symmetry were provided by Morris ${ }^{9}$ in tabular form, and are reproduced here in Table I

As can be noted from Equation (2) above, only three of the texture coefficients, $W_{400}, W_{420}$, and $W_{440}$, enter into the weighting function for averaging the elastic properties of the textured polycrystalline aggregates. This means materials having quite different textures could have the same elastic properties if the three texture coefficients, $W_{4} 00$, $\mathrm{W}_{420}$, and $\mathrm{W}_{440}$, were the same. It is, therefore, clear that one cannot determine the texture of a polycrystalline material by measuring its elastic constants, even though the 
TABLE I

$\overline{\mathrm{T}}_{i j k l}$ for Cubic Crystal symmetry (After Morris ${ }^{9}$ )

\begin{tabular}{|c|c|c|c|c|}
\hline A & $\underline{000}$ & 400 & $\underline{420}$ & 440 \\
\hline$\overline{\mathrm{T}}$ & $\underline{B_{0} X}$ & $\underline{B_{4} X}$ & $\underline{B_{5} X}$ & 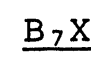 \\
\hline 1111 & 18 & 72 & -24 & \\
\hline 1112 & 12 & -72 & 24 & 24 \\
\hline 2211 & 18 & 72 & 24 & \\
\hline 2212 & 12 & -72 & -24 & -24 \\
\hline 3311 & 18 & 192 & --- & \\
\hline 3312 & 12 & -192 & --- & \\
\hline 1211 & 6 & 24 & --- & -24 \\
\hline 1212 & 24 & -24 & --- & 27 \\
\hline 1311 & 6 & -96 & 24 & - \\
\hline 1312 & 24 & 96 & -24 & - \\
\hline 2311 & 6 & -96 & -24 & - \\
\hline 2312 & 24 & 96 & 24 & - \\
\hline 4412 & -6 & 96 & 24 & - \\
\hline 5512 & -6 & 96 & -24 & . \\
\hline 6612 & -6 & -24 & --- & 24 \\
\hline
\end{tabular}

where $\mathrm{B}_{0}=1 / 30, \mathrm{~B}_{4}=\sqrt{2} / 840, \mathrm{~B}_{5}=\sqrt{5} / 210$ and $B_{7}=\sqrt{35} / 420$. The table contains fifteen $\bar{T}_{i j k l}$, the remaining twelve are given by relations of the form $\overline{\mathrm{T}}_{1244}=2 \overline{\mathrm{T}}_{1112,}, \overline{\mathrm{T}}_{1244}=2 \overline{\mathrm{T}}_{6612,}, \overline{\mathrm{T}}_{4411}=$ $\overline{\mathrm{T}}_{2311,}$, and $\mathrm{T}_{6644}=\mathrm{T}_{1212}+\mathrm{T}_{6612}$.

elastic constants of the material will be affected by its texture.

From the elastic stiffness constants of an iron single crystal and the texture coefficients of the phosphorus-steel sheets compiled in Table II, the weighted Voigt averages were calculated by using Equations 1 and 2 with appropriate values of $\bar{T}_{i j k \ell}$ taken from Table $I$. The results are summarized in Table III for both the cold-rolled and the annealed sheets of the steel. For the benefit of those who are unfamiliar with this kind of problem, an example showing the actual calculations is provided in Item 1 of the Appendix.

\section{The Weighted Reuss Average}

The Reuss averaging procedure is based on the assumption that the stress is uniform throughout the aggregate. The calculations will be performed for the average elastic compliances, $\bar{s}_{i j k \ell}$, of the aggregate by using the elastic compliances of the single crystal. Because the definitions for the two-index notation of some of the compliances, sij, are a little different from those of the stiffnesses, $c_{i j}$ ' (see Reference 19 for details), the appropriate expression for averaging the elastic compliance $\bar{s}_{1111}$, in contrast to Equation (la) for $\bar{c}_{11}$, will be as follows: 
TABLE II

Elastic Constants of Iron Single Crystal and Texture Coefficients of Cold-Rolled and Annealed Sheets of Phosphorus Steel

Elastic Constants of Iron Single Crystal 20

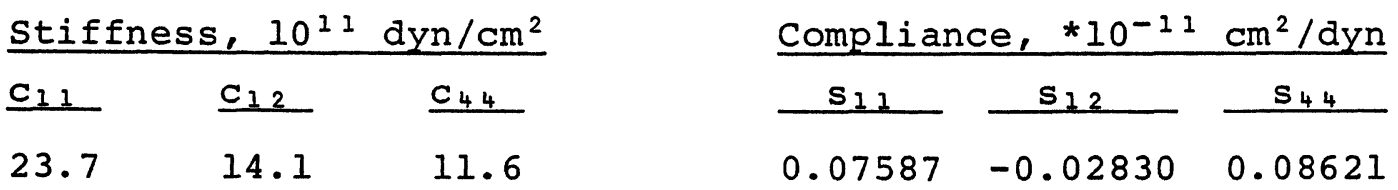

Texture Coefficients of Phosphorus Steel sheet ${ }^{1}$

\begin{tabular}{|c|c|c|c|c|}
\hline Sample & $\mathrm{W}_{400}$ & & $\mathrm{~W}_{420}$ & $\mathrm{~W}_{440}$ \\
\hline Cold Rolled & $-3.3491 \times$ & $\times 10^{-3}$ & $-2.4172 \times 10^{-3}$ & $-5.5361 \times 10^{-3}$ \\
\hline Annealed & $-11.5013 \times$ & $\times 10^{-3}$ & $-2.9016 \times 10^{-3}$ & $-0.0346 \times 10^{-3}$ \\
\hline
\end{tabular}

TABLE III

The Texture-Weighted Averages of the Elastic Stiffness Constants of the Phosphorus Steel

(in units of $10^{11} \mathrm{dyn} / \mathrm{cm}^{2}$ or $10^{10} \mathrm{~N} / \mathrm{m}^{2}$ )

$\begin{array}{llllllllllllllllllllll}\text { Average } & \bar{c}_{11} & \bar{c}_{22} & \bar{c}_{33} & \bar{c}_{23} & \bar{c}_{31} & \bar{c}_{12} & \bar{c}_{55} & \bar{c}_{66}\end{array}$

Cold-Rolled sheet

$\begin{array}{llllllllll}\text { Voigt } & 30.03 & 30.69 & 29.72 & 10.76 & 11.42 & 10.45 & 8.26 & 8.92 & 7.95 \\ \text { Reuss } & 28.18 & 29.00 & 27.81 & 11.65 & 12.46 & 11.27 & 6.84 & 7.44 & 6.59 \\ \text { Hill } & 29.11 & 29.85 & 28.77 & 11.20 & 11.94 & 10.86 & 7.55 & 8.18 & 7.27\end{array}$

Annealed Sheet

$\begin{array}{lrllllllll}\text { Voigt } & 29.50 & 30.29 & 31.14 & 9.98 & 10.78 & 11.62 & 7.48 & 8.28 & 9.12 \\ \text { Reuss } & 26.76 & 27.76 & 28.79 & 10.30 & 11.29 & 12.34 & 6.24 & 6.86 & 7.65 \\ \text { Hill } & 28.13 & 29.03 & 29.97 & 10.14 & 11.04 & 11.98 & 6.86 & 7.57 & 8.39\end{array}$

$\bar{s}_{1111}=\bar{T}_{1111} S_{1111}+\bar{T}_{1112} S_{1122}+\bar{T}_{1144} S_{2323}$

Similar expressions for averaging the other elastic compliances are given in Item 2 of the Appendix. 
From the elastic compliances of an iron single crystal provided in Table II*, the weighted Reuss averages of the elastic compliances were calculated for the cold-rolled and the annealed sheets. For direct comparison with the Voigt averages, the elastic compliances of the Reuss averages were converted into the stiffness constants by inversion, and the results are summarized in Table III. The Voigt averages are invariably higher than the Reuss averages, in agreement with the analysis of Hill.14 An example of these calculations for the Reuss averages is also provided in the Appendix (Item 2).

The Weighted Hill Average

The Hill averages can simply be taken as the mean values of the Voigt and Reuss averages, namely

$$
\begin{array}{r}
\bar{s}_{i j k l}(H)=\frac{1}{2}\left[\bar{s}_{i j k \ell}(V)+\bar{s}_{i j k \ell}(R)\right] \\
\text { or } \bar{c}_{i j k l}(H)=\frac{1}{2}\left[\bar{c}_{i j k \ell}(V)+\bar{c}_{i j k \ell}(R)\right] \\
\text { providing }\left(\bar{s}_{p q}(V)\right)=\left(\bar{c}_{p q}(V)\right)^{-1}
\end{array}
$$

where (H), (V) and (R) denote Hill, Voigt, and Reuss values respectively. The Hill averaged stiffness constants of the cold-rolled and the annealed sheets of the phosphorus steel are also listed in Table III, together with the averages of Voigt and Reuss.

\section{COMPARISON BETWEEN CALCULATED AND MEASURED ELASTIC PROPERTIES}

To check the validity of these texture-weighted calculations of the elastic constants, the Young's modulus along various directions in the plane of the sheet was calculated from the averaged stiffness constants summarized in Table III and compared with experimentally determined values. The Young's modulus, E, was determined ultrasonically by measuring the resonant frequency of a long narrow strip-specimen ( 4 by 0.25 by 0.030 in., or 101 by 6.4 by $0.8 \mathrm{~mm}$ ) when a longitudinal wave was propagated along the length of the

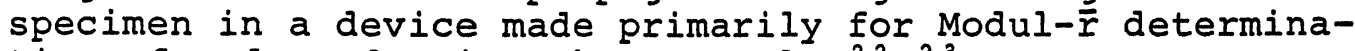
tions for deep-drawing sheet steels. 22,23 expression

The Young's modulus, $E$, was calculated from the

$$
E=\rho(2 L f)^{2}
$$

where $\rho$ is the density of the steel, $L$ is the length of the specimen, and $f$ is the resonant frequency. This technique was used earlier by Alers and $\mathrm{Liu}^{24,25}$ for studying the

\footnotetext{
*The elastic compliances of iron single crystal, $s_{11}=s_{1111}$, $s_{12}=s_{1122}$, and $s_{44}=4 s_{2323}$, can be obtained by matrix inversion of corresponding $c_{i j}$, the elastic stiffnesses, or by using the relations between $c_{i j}$ and $s_{i j}$ as given, for example, in Reference 21 .
} 
cold-rolling textures of copper and brass. Results from these measurements on duplicate specimens of cold-rolled and of annealed sheets of the steel are summarized in Table IV.

\section{TABLE IV}

Young's Modulus of Cold-Rolled and of Annealed Sheets for a Phosphorus Steel as Determined from the Ultrasonic Measurements

Angle from

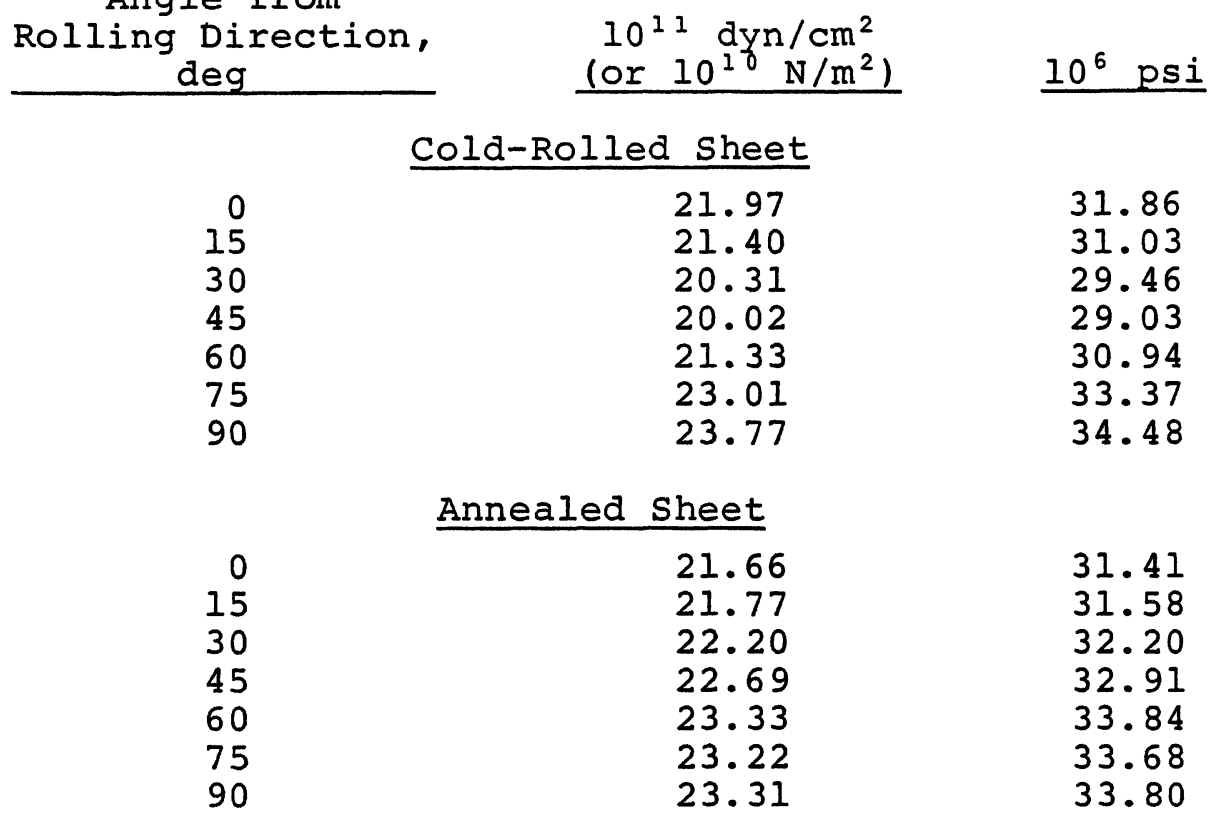

Because $\mathrm{E}=1 / \mathrm{s}_{11}$, the calculation of the Young's modulus along the various directions in the plane of the sheet will essentially amount to first calculating the elastic compliance constants $s_{11}$ for the various directions in the plane of the sheet and then taking the reciprocal values of these constants. When the corresponding stiffness constants, $c_{i j}$, are known, the values of the $s_{i j}$ can be calculated by matrix inversion, as already shown in Item 2 of the Appendix. The calculated averages of the stiffness constants, as summarized in Table III, had the index notations with the specimen or physical coordinate axes xyz (see Figure 3 of Reference 1) as the common reference frame. The right-hand axis system was consistently followed in indexing the elastic constants. For example, 1 refers to the $x$-axis or the rolling direction of the sheet, 2 to the $y$-axis or the transverse direction of the sheet, and 3 to the z-axis or the normal direction of the sheet.

To calculate the elastic properties (such as the Young's modulus) along the various directions in the plane of the 
sheet from the elastic constants given in Table III, a corresponding rotation of the specimen coordinate system would have to be introduced. In other words, the elastic stiffness constants shown in Table III must be transformed from the initial specimen coordinate system xyz to the new coordinate system $x^{\prime} y^{\prime} z$ ' by a rotation of $\theta$ degrees around the $z$-axis, or the normal direction, of the sheet. Such axis rotation is illustrated in Figure 1 , and the transformation between the two sets of coordinate axes can be conveniently specified by the scheme,

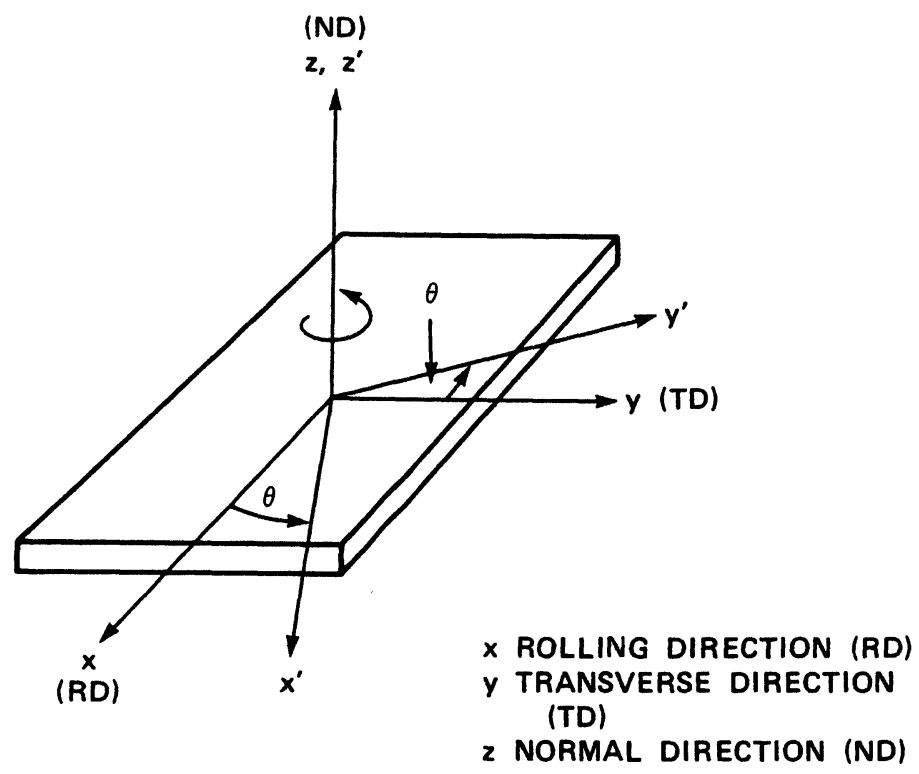

Figure 1. Rotation of the specimen coordinate system around the z-axis or the normal direction of the sheet.

\begin{tabular}{c|ccc}
\multicolumn{1}{c}{} & $\mathrm{x}$ & $\mathrm{y}$ & $\mathrm{z}$ \\
\cline { 2 - 4 } $\mathrm{x}^{\prime}$ & $\mathrm{l}_{1}$ & $\mathrm{~m}_{1}$ & $\mathrm{n}_{1}$ \\
$\mathrm{z}^{\prime}$ & $l_{2}$ & $\mathrm{~m}_{2}$ & $\mathrm{n}_{2}$ \\
$l_{3}$ & $\mathrm{~m}_{3}$ & $\mathrm{n}_{3}$
\end{tabular}

where $l_{i}, m_{i}$, and $n_{i}$ are the elements of the rotation matrix. For rotations around the z-axis or the normal direction of the sheet, as shown in Figure 1 , the rotation matrix elements are as follows:

$$
\left(\begin{array}{lll}
l_{1}=\cos \theta & \mathrm{m}_{1}=\sin \theta & \mathrm{n}_{1}=0 \\
l_{2}=-\sin \theta & \mathrm{m}_{2}=\cos \theta & \mathrm{n}_{2}=0 \\
l_{3}=0 & \mathrm{~m}_{3}=0 & \mathrm{n}_{3}=1
\end{array}\right)
$$


The numerical values of the stress or the strain components depend on the particular coordinate system that is being used as the reference frame. By using the relations between the stress components referred to the initial and the transformed coordinate systems (see the derivation and formulation of these relations by Zener ${ }^{26}$ ) and the relations between stress and strain in the generalized form of Hook's law, a set of general equations for calculating the stiffness constants, c cij' with reference to the rotated coordinate systems were obgtained. These equations are provided in

Item 3 of the Appendix.

As can be noted from these equations, there are 13 nonzero stiffness constants, $c_{i j}^{\prime}$, with reference to the rotated coordinate system. These stiffness constants, $c_{i j}^{\prime}$, are related to corresponding compliance constants, sij, by matrix inversion, as follows:

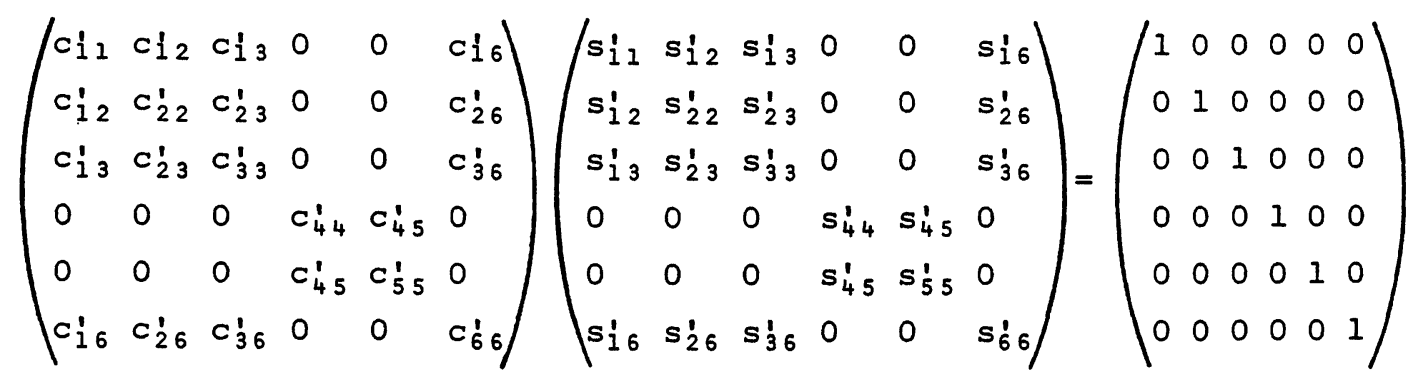

Calculations for the $c_{i j}^{\prime}$ constants for $\theta=0,15,30$, $45,60,75$, and 90 degrees according to the equations given in Item 3 of the Appendix and the matrix inversions as formulated in Equation (9) above were performed by computer. The values of $s_{i}$ and the Young's modulus, $E=1 / s_{1}^{\prime}$, as a function of $\theta$ are listed in Table $V$. These calculated values of $E$, together with the Young's modulus determined experimentally (Table IV), are plotted in Figure 2 for a direct comparison of the relative validity of the averaging methods incorporated with texture-weighting functions. It is clear that the $\mathrm{Hill}$ averages are very close to the experimentally determined results, whereas those of Voigt and Reuss represent, respectively, the upper and lower limits. The present experimentally determined results for both the as-cold-rolled and the fully annealed sheets of phosphorus steel thus support Hill's analysis, ${ }^{4}$ as do the results on other sheet materials. $8,9,11$

For sheet in the annealed condition, the angular variations of Young's modulus in the plane of the sheet for the present phosphorus steel are distinctly different from those for the aluminum-killed deep-drawing steels analyzed by Bunge and Roberts. ${ }^{1}$ As shown by the upper portion of Figure 2 , the Young's modulus of the phosphorus steel increased with the angle to the rolling direction, reaching a maximum at the transverse direction $(\theta=90$ degrees). For the aluminumkilled steels, the Young's modulus attained a maximum at 
TABLE V

Summary of the Calculated Elastic Compliance Constants silil and the Young's Modulus, E, as a Function

of the Rotation Angle, $\theta$

\begin{tabular}{|c|c|c|c|c|c|c|}
\hline \multirow{2}{*}{$\begin{array}{c}\theta \\
\left(\operatorname{deg}^{2}\right) \\
\end{array}$} & \multicolumn{2}{|c|}{ Voigt } & \multicolumn{2}{|c|}{ Reuss } & \multicolumn{2}{|c|}{$\mathrm{Hill}$} \\
\hline & $s_{i 21}$ & $\underline{E}$ & $s_{1121}$ & $E$ & $s_{111}^{\prime}$ & $\underline{E}$ \\
\hline \multicolumn{7}{|c|}{ Cold-Rolled sheet } \\
\hline $\begin{array}{r}0 \\
15 \\
30 \\
45 \\
60 \\
75 \\
90\end{array}$ & $\begin{array}{l}0.04140 \\
0.04286 \\
0.04569 \\
0.04682 \\
0.04480 \\
0.04132 \\
0.03962\end{array}$ & $\begin{array}{l}24 \cdot 15 \\
23 \cdot 33 \\
21 \cdot 89 \\
21 \cdot 36 \\
22 \cdot 32 \\
24 \cdot 20 \\
25.24\end{array}$ & $\begin{array}{l}0.04744 \\
0.04949 \\
0.05345 \\
0.05496 \\
0.05196 \\
0.04690 \\
0.04445\end{array}$ & $\begin{array}{l}21.08 \\
20.21 \\
18.71 \\
18.20 \\
19.25 \\
21.32 \\
22.50\end{array}$ & $\begin{array}{l}0.04417 \\
0.04589 \\
0.04922 \\
0.05051 \\
0.04806 \\
0.04388 \\
0.04185\end{array}$ & $\begin{array}{l}22.64 \\
21.79 \\
20.32 \\
19.80 \\
20.81 \\
22.79 \\
23.89\end{array}$ \\
\hline \multicolumn{7}{|c|}{ Annealed Sheet } \\
\hline $\begin{array}{r}0 \\
15 \\
30 \\
45 \\
60 \\
75 \\
90\end{array}$ & $\begin{array}{l}0.04291 \\
0.04277 \\
0.04240 \\
0.04188 \\
0.04135 \\
0.04095 \\
0.04081\end{array}$ & $\begin{array}{l}23.31 \\
23.38 \\
23.59 \\
23.88 \\
24.19 \\
24.22 \\
24.50\end{array}$ & $\begin{array}{l}0.05136 \\
0.05088 \\
0.04974 \\
0.04858 \\
0.04788 \\
0.04766 \\
0.04764\end{array}$ & $\begin{array}{l}19.47 \\
19.65 \\
20.11 \\
20.59 \\
20.89 \\
20.98 \\
20.99\end{array}$ & $\begin{array}{l}0.04671 \\
0.04643 \\
0.04573 \\
0.04493 \\
0.04433 \\
0.04400 \\
0.04391\end{array}$ & $\begin{array}{l}21.41 \\
21.54 \\
21.87 \\
22.26 \\
22.56 \\
22.73 \\
22.77\end{array}$ \\
\hline Units: & $\begin{array}{l}s_{1111}^{\prime} \\
E\end{array}$ & $\begin{array}{l}\text { in } \mathrm{cm}^{2} / 1 \\
\text { in } 10^{11}\end{array}$ & $\begin{array}{l}1 \text { dyn or } \\
\mathrm{n} / \mathrm{cm}^{2} \text { or }\end{array}$ & $\begin{array}{l}\mathrm{m}^{2} / 10^{10} \\
10^{10} \mathrm{~N} / \mathrm{m}^{2}\end{array}$ & & \\
\hline
\end{tabular}

approximately the diagonal direction $(\theta=45$ degrees). 11 This observation is consistent with the results reported earlier that the annealing texture of phosphorus steels is distinctly different from that of aluminum-killed steels. I

This demonstrates the validity of the use of textureweighted calculations to establish the elastic properties of sheet steels and suggests that this technique may be applied to the calculation of other physical properties and certain orientation dependent mechanical properties from the orientation distribution function of the crystallites. Conversely, measurement of elastic moduli in different directions can provide information about the texture and drawability of sheet steel (use of MODUL- $\bar{r}$ to predict formability) but such measurements are not sufficient to establish the complete details of the preferred orientation of the sheet.

\section{SUMMARY}

1. The elastic properties of as-cold-rolled and of fully annealed sheets of a phosphorus steel having high normal plastic anisotropy ( $r$ value) and low planar plastic an- 


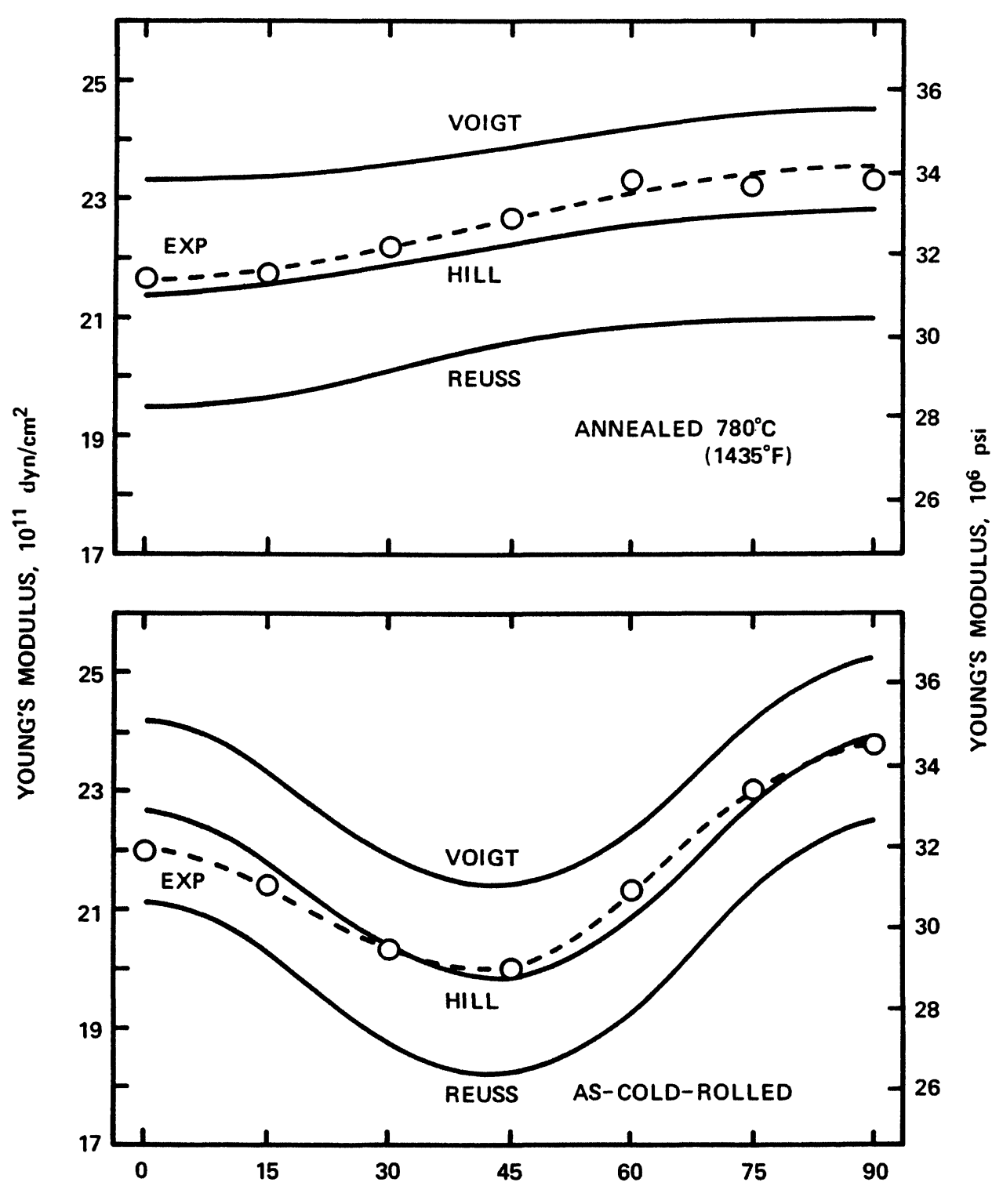

ANGLE TO ROLLING DIRECTION, 0 , deg

Figure 2. Comparison of the calculated and the experimentally determined Young's modulus of the cold-rolled and the annealed sheets of phosphorus steel along the various directions in the plane of the sheet.

isotropy ( $\Delta r$ value) were calculated by using three different averaging schemes proposed respectively by Voigt, Reuss, and Hill, and texture weighting functions as formulated by Morris. For the benefit of those scientists or engineers who are unfamiliar with this kind of problem and wish to conduct similar calculations in their own research, the calculation procedures are described in detail, and examples of calculations are provided. The calculated values of Young's modulus along 
the various directions in the plane of the sheet were compared with those determined experimentally.

2. Consistent with Hill's analysis and with the results reported for other sheet materials, the calculated Voigt averages are somewhat too high, and the Reuss averages too low, in comparison with experimentally determined values.

The Hill averages are very close to the measured values.

3. The angular variations of Young's modulus in the plane of the annealed sheet of the present phosphorus steel exhibit features distinctly different from those of the aluminum-killed deep-drawing sheet steels. This is consistent with earlier results, indicating that the annealing texture of the phosphorus steels is distinctly different from that of aluminum-killed steels.

4. The present results lend further support to the validity of the texture-weighted calculations for the elastic properties of sheet materials and demonstrate the potential for using these techniques to determine other physical properties and certain orientation-dependent mechanical properties.

\section{ACKNOWLEDGMENT}

The author wishes to thank P. R. Morris of the Armco Inc. and $Y$. T. Chou of Lehigh University for frequent consultations during the course of this work, and $R$. W. Whitmore of this Laboratory for computer programming for matrix inversion calculations. Comments and critical reading of the manuscript by L. F. Porter and by O. Richmond were particularly appreciated.

\section{REFERENCES}

1. H. Hu, Texture Cryst. Sol., 4, 13 (1980).

2. R. J. Roe, J. Appl. Phys., 36, 2024 (1965).

3. R. J. Roe, J. Appl. Phys., 37, 2069 (1966).

4. H. J. Bunge, Zeit. Metallk., 56, 872 (1965).

5. H. J. Bunge, Mathematische Methoden der Texturanalyse (AkademieVerlag, Berlin, 1969).

6. H. J. Bunge, R. Ebert and F. Ginther, Phys. Sta. Sol., 31, 565 (1969).

7. J. S. Kallend and G. J. Davies, J. Inst. Met., 98, 242 (1970).

8. J. S. Kallend and G. J. Davies, J. Inst. Met., 5,257 (1971).

9. P. R. Morris, Int. J. Eng. Sci., 8, 49 (1970).

10. P. R. Morris, Met. Trans., $2,194 \overline{9}$ (1971).

11. H. J. Bunge and W. T. Roberts, J. Appl. Cryst., 2, 116 (1969).

12. W. Voigt, Lehrbuch der Kristallphysik (Teubner Verlag, Leipzig, 1928), p. 739.

13. A. Reuss, Zeit. angew. Math. u. Mech., 9, 49 (1929).

14. R. Hill, Phys. Soc. Proc. (London), 65, 349 (1952).

15. D. H. Chung and W. R. Buessem, J. Appl. Phys., 38, 2535 (1967).

16. E. Kröner, Zeit. Phys., 151, 504 (1958).

17. H. J. Bunge, Krist. u. Tech., 3, 431 (1968).

18. P. R. Morris, J. Appl. Phys., 40,447 (1969).

19. J. F. Nye, Physical Properties of Crystals (Clarendon Press, Oxford, 1957), p. 135. 
20. W. P. Mason, Piezoelectric Crystals and Their Application to Ultrasonics (Van Nostrand, 1950), p. 417.

21. J. F. Nye, Physical Properties of Crystals (Clarendon Press, Oxford, 1957), p. 147.

22. C. A. Stickels and P. R. Mould, Met. Trans., 1, 1303 (1970).

23. P. R. Mould and T. E. Johnson, Jr., Sheet Metal Ind., 50, 328, 348 (1973).

24. G. A. Alers and Y. C. Liu, "Calculation of Elastic Anisotropy in Rolled Sheet," Trans. TMS-AIME, 236, 482 (1966).

25. Y. C. Liu and G. A. Alers, Trans. TMS-AIME, 236, 489 (1966).

26. C. Zener, Elasticity and Anelasticity of Metals (University of Chicago Press, 1956), p. 7.

\section{APPENDIX}

1. Calculation of the Voigt Average of the Elastic Stiffness Constants

For the $\bar{c}_{11}$ of the cold-rolled sheet:

Take Equation $(1) *,\left[\bar{c}_{i j}\right]=\left[\overline{\mathbf{T}}_{i j k \ell}\right]\left[\mathrm{c}_{k \ell}\right]$

$$
\bar{C}_{11}=\bar{T}_{1111} C_{11}+\bar{T}_{1112} C_{12}+\bar{T}_{1144} \dot{C}_{44}
$$

where $\bar{c}_{11}=\bar{c}_{1111}, c_{11}=c_{1111}, c_{12}=c_{1122}, c_{44}=c_{2323}$. Take Equation (2), and data from Tables I and II:

$$
\begin{aligned}
& \overline{\mathrm{T}}_{1 j k l}= A_{000}+4 \pi^{2}\left(\mathrm{~A}_{400} \mathrm{~W}_{400}+\mathrm{A}_{420} \mathrm{~W}_{420}+\mathrm{A}_{440} \mathrm{~W}_{440}\right) \\
& \overline{\mathrm{T}}_{1111}= \frac{18}{30}+4 \pi^{2}\left[\frac{72 \sqrt{2}}{840}\left(-3.3491 \times 10^{-3}\right)\right. \\
&+\left.\frac{-24 \sqrt{5}}{210}\left(-2.4172 \times 10^{-3}\right)+\frac{24 \sqrt{35}}{420}\left(-5.5361 \times 10^{-3}\right)\right] \\
&= 0.53447 \\
& \overline{\mathrm{T}}_{1112}= \frac{12}{30}+4 \pi^{2}\left[\frac{-72 \sqrt{2}}{840}\left(-3.3491 \times 10^{-3}\right)\right. \\
&\left.+\frac{24 \sqrt{5}}{210}\left(-2.4172 \times 10^{-3}\right)+\frac{-24 \sqrt{35}}{420}\left(-5.5361 \times 10^{-3}\right)\right] \\
&= 0.46553 \\
& \overline{\mathrm{T}}_{1144}= 2 \overline{\mathrm{T}}_{1112}=0.93106 \\
& \text { Hence, } \overline{\mathrm{C}}_{11}=(0.53447)(23.7)+(0.46553)(14.1) \\
& \quad+(0.93106)(11.6) \\
&=30.03 \times 10^{11} \mathrm{dyn} / \mathrm{cm}^{2} .
\end{aligned}
$$

* See text equations. 
2. Calculation of the Reuss Averages of the Elastic Compliances

To avoid confusion and possible mistake in calculation, the four-index notation should be used for the compliances. Thus

$$
\begin{aligned}
& \bar{s}_{1111}=\bar{T}_{1111} s_{1111}+\bar{T}_{1112} s_{1122}+\bar{T}_{1144} s_{2323} \\
& \bar{s}_{2221}=\bar{T}_{2111} s_{1111}+\bar{T}_{2212} s_{1122}+\bar{T}_{2244} s_{2323} \\
& \bar{s}_{3333}=\bar{T}_{3311} s_{1111}+\bar{T}_{3312} s_{1122}+\bar{T}_{3344} s_{2323} \\
& \bar{s}_{2233}=\bar{T}_{2311} s_{1111}+\bar{T}_{2312} s_{1122}+\bar{T}_{2344} s_{2323} \\
& \bar{s}_{3311}=\bar{T}_{3111} s_{1111}+\bar{T}_{3112} s_{1122}+\bar{T}_{3144} s_{2323} \\
& \bar{s}_{1122}=\bar{T}_{1211} s_{1111}+\bar{T}_{1212} s_{1122}+\bar{T}_{1244} s_{2323} \\
& \bar{s}_{2323}=\bar{T}_{4411} s_{1111}+\bar{T}_{4412} s_{1122}+\bar{T}_{4444} s_{2323} \\
& \bar{s}_{3131}=\bar{T}_{5511} s_{1111}+\bar{T}_{5512} s_{1122}+\bar{T}_{5544} s_{2323} \\
& \bar{s}_{1212}=\bar{T}_{6611} s_{1111}+\bar{T}_{6612} s_{1122}+\bar{T}_{6644} s_{2323}
\end{aligned}
$$

The corresponding averaged stiffnesses can be obtained from the last three compliances, namely $\bar{s}_{2323,} \bar{s}_{3131}$ and $\bar{s}_{1212}$, by simple inversion, such as

$$
\begin{aligned}
& \bar{c}_{44}=\bar{c}_{2323}=1 / \bar{s}_{44}=1 / 4 \bar{s}_{2323} \\
& \bar{c}_{55}=\bar{c}_{3131}=1 / \bar{s}_{55}=1 / 4 \bar{s}_{3131} \\
& \bar{c}_{66}=\bar{c}_{1212}=1 / \bar{s}_{66}=1 / 4 \bar{s}_{1212}
\end{aligned}
$$

For the other six compliances, namely $\bar{s}_{1111}, \bar{s}_{2222}$, $\bar{s}_{3333}, \bar{s}_{2233}, \bar{s}_{3311}$, and $\bar{s}_{1122}$, the corresponding averaged stiffness constants $\bar{c}_{1111}, \bar{c}_{2222,} \bar{c}_{3333}, \bar{c}_{2233}, \bar{c}_{3311}$, and $\overline{\mathbf{c}}_{1122}$ (in two-index notation, they are $\bar{c}_{11}, \overline{\mathbf{c}}_{22}, \overline{\mathbf{c}}_{33}, \overline{\mathbf{c}}_{23}$, $\bar{c}_{31}$, and $\bar{c}_{12}$, respectively) have to be obtained by matrix inversion as follows:

$\left(\begin{array}{lll}\bar{s}_{11} & \bar{s}_{11} & \bar{s}_{11} \\ \bar{s}_{22} & \bar{s}_{22} & \bar{s}_{22} \\ \bar{s}_{33} & \bar{s}_{33} & \bar{s}_{33}\end{array}\right) \times\left(\begin{array}{lll}\bar{c}_{11} & \bar{c}_{11} & \bar{c}_{11} \\ \bar{c}_{22} & \bar{c}_{22} & \bar{c}_{22} \\ \bar{c}_{33} & \bar{c}_{33} & \bar{c}_{33}\end{array}\right)=\left(\begin{array}{lll}1 & 0 & 0 \\ 0 & 1 & 0 \\ 0 & 0 & 1\end{array}\right)$

That is, $\left(\bar{c}_{i j}\right)=\left(\bar{s}_{i j}\right)^{-1}$

$$
\bar{c}_{i j}=\bar{c}_{j i} \text {, and } \bar{s}_{i j}=\bar{s}_{j i}
$$


3. Equations for Calculating the Elastic Stiffness Constants, $c_{i j k l}^{\prime}$, with Reference to the Rotated Coordinate System

Using matrix notation for simplifying the expressions, the equations for calculating the elastic stiffness constants, c!' of the strip specimens cut along a direction $\theta$ degrees from the rolling direction on the plane of the rolled sheet from the elastic stiffness constants, $c_{i j}$, of the sheet with reference to the initial coordinate axes xyz (see Figure 1 of the text for the geometrical relations between the initial coordinate axes $x y z$ and the rotated coordinate axes $\left.x^{\prime} y^{\prime} z^{\prime}\right)$ are given below:

$$
\begin{aligned}
& c_{11}^{\prime}=\cos ^{4} \theta c_{11}+\sin ^{4} \theta c_{22}+2 \sin ^{2} \theta \cos ^{2} \theta\left(c_{12}+2 c_{66}\right) \\
& c_{12}^{\prime}=\cos ^{2} \theta \sin ^{2} \theta\left(c_{11}+c_{22}-4 c_{66}\right)+\left(\sin ^{4} \theta+\cos ^{4} \theta\right) c_{12} \\
& c_{13}=\cos ^{2} \theta c_{13}+\sin ^{2} \theta c_{23} \\
& \mathrm{Ci}_{4}=0 \\
& c_{15}=0 \\
& c_{16}^{\prime}=\cos \theta \sin \theta\left[\cos ^{2} \theta\left(c_{12}-c_{11}+2 c_{66}\right)+\sin ^{2} \theta\left(c_{22}-c_{12}-2 c_{66}\right)\right] \\
& c_{22}^{\prime}=\sin ^{4} \theta c_{11}+\cos ^{4} \theta c_{22}+2 \sin ^{2} \theta \cos ^{2} \theta\left(c_{12}+2 c_{66}\right) \\
& c_{23}^{1}=\sin ^{2} \theta c_{13}+\cos ^{2} \theta c_{23} \\
& c_{24}^{\prime}=0 \\
& C_{25}^{\prime}=0 \\
& c_{26}^{\prime}=\sin \theta \cos \theta\left[\sin ^{2} \theta\left(c_{12}-c_{11}+2 c_{66}\right)+\cos ^{2} \theta\left(c_{22}-c_{12}-2 c_{66}\right)\right] \\
& c_{33}^{\prime}=C_{33} \\
& \mathrm{C}_{34}^{\prime}=0 \\
& c_{35}^{\prime}=0 \\
& c_{36}^{\prime}=\cos \theta \sin \theta\left(c_{32}-c_{31}\right)=\cos \theta \sin \theta\left(c_{23}-c_{13}\right) \\
& c_{44}^{1}=\cos ^{2} \theta c_{44}+\sin ^{2} \theta c_{55} \\
& c_{45}^{\prime}=\cos \theta \sin \theta\left(c_{44}-c_{55}\right) \\
& c_{46}^{\prime}=0 \\
& c_{55}^{\prime}=\sin ^{2} \theta c_{44}+\cos ^{2} \theta c_{55} \\
& c_{5}^{\prime} 6=0 \\
& c_{66}^{\prime}=\cos ^{2} \theta \sin ^{2} \theta\left(c_{11}-2 c_{12}+c_{22}\right)+\left(\cos ^{2} \theta-\sin ^{2} \theta\right)^{2} c_{66}
\end{aligned}
$$


Note: Because of symmetry, $c_{i j}^{\prime}=c_{j i}^{\prime}$ :

$$
\begin{array}{lll}
c_{12}^{\prime}=c_{21}^{\prime} & c_{45}^{\prime}=c_{54}^{\prime} & c_{61}^{\prime}=c_{16}^{\prime} \\
c_{13}^{\prime}=c_{31}^{\prime} & c_{46}^{\prime}=c_{64}^{\prime} & c_{62}^{\prime}=c_{26}^{\prime} \\
c_{23}^{\prime}=c_{32}^{\prime} & c_{56}^{\prime}=c_{65}^{\prime} & c_{63}^{\prime}=c_{36}^{\prime}
\end{array}
$$

Correspondence between matrix and tensor notations ${ }^{19}$ : Matrix notation $\quad \begin{array}{llllll}1 & 2 & 3 & 4 & 5 & 6\end{array}$

Tensor notation $11 \quad 22 \quad 33 \quad 23,32 \quad 31,13 \quad 12,21$

No numerical factors are involved for the change of notations for elastic stiffness constants (the c's).

However, for the elastic compliances (the s's), factors of 2 and 4 are introduced as follows:

$$
\begin{aligned}
s_{i j k l} & =s_{m n} \text { when } m \text { and } n \text { are } 1,2 \text {, or } 3, \\
2 s_{i j k l} & =s_{m n} \text { when either } m \text { or } n \text { are } 4,5 \text {, or } 6 \text {, } \\
4 s_{i j k l} & =s_{m n} \text { when both } m \text { and } n \text { are } 4,5 \text {, or } 6 .
\end{aligned}
$$

